\title{
Performance of THM behavior of sand-bentonite mixtures considering thermal effect
}

\author{
Tomoyoshi Nishimura $^{{ }^{*}}$ and Junichi Koseki $^{2}$ \\ ${ }^{1}$ Department of Civil Engineering, Ashikaga University, Japan \\ ${ }^{2}$ Department of Civil Engineering, The University of Tokyo, Japan
}

\begin{abstract}
This study presented the overview on the behavior of bentonite-sand mixture used in high level radioactive waste disposal. Both unconfined compression test and triaxial compression test were conducted out that unsaturated-saturated bentonite-sand samples were applied temperature effect below 100 degrees Celsius. Unconfined compressive strength was determined with various temperatures and different relative humidity for unsaturated bentonite-sand specimens, so the growing of pore pressure due to heating was most significant for interpretation to THM behaviour of artificial barrier system compositing bentonite materials.
\end{abstract}

\section{Introduction}

Compacted bentonite with high swelling performance is included in buffer and backfill materials in high-level radioactive waste (HLW). Extremely low hydraulic conductivity, strong swelling pressure, complex macromicro structure, high water retention properties related to barrier made of bentonite (Delage et al., (2010) [1]). It is required to restrict disseminate of radionuclides from the waste metal package (Wersin et al., (2007) [2]). Severe problems such these properties, however, were produced by thermally-induced effects that the decay heat from waste caused. The influence of high temperature on bentonite properties is one of significant targets on thermos-hydro-mechanical (THM) evolution in the swelling material barrier, should be investigated adequate. The bentonite with sand barrier, initially, under unsaturated condition is subjected to some thermal, hydraulic gradients and high external loading, because of its expansive performance is expected to swell close to the host rock as result of the water inflow (Akesson et al., (2009) [3]). Previous studies describe the laboratory experiments for thermos-hydro-mechanical-chemical (THMC) evolution in the bentonite barrier (Tang et al., (2008) [4]). Some of experimental investigations referred to THM numerical simulations of bentonite barriers with higher temperature (i.e., above 100 degrees Celsius) and applied various temperature gradients. Akesson et al., (2009) [3] described the behavior of an unsaturated expansive clay (MX-80 bentonite) when the bentonite was subjected to a thermal gradient under confined conditions in the context of engineer barriers for radioactive waste emplacement. The research performed to verify numerical simulation of the thermos-hydromechanical (THM) process involved the evolution of temperature, relative humidity, pore pressure and stresses. Actually, laboratory experimental works below 100 degrees Celsius widely have been conducted out in the previous studies (Villar \& Lloret (2004) [5]). Some papers have presented the identification and characterization of disposal concepts for swelling materials subjected to heating.

\section{Purpose of this study}

This study presented the overview on the behavior of bentonite-sand mixture used in high level radioactive waste disposal. Both unconfined compression test and triaxial compression test were conducted out that unsaturated-saturated bentonite-sand samples were applied temperature below 100 degrees Celsius. On the basis of the traditional heating system, the temperature triaxial compression test system was developed in this study. The newly developed system could meet the requirement of various temperatures in a range from 20 to 100 degrees Celsius. Unconfined compressive strength and compressive strength having various lateral confining pressures are significant importance for analysis and design of waste disposal barrier. The main objections of this study are to investigate the influence of thermal effect on behavior of unsaturated-saturated bentonite-sand sample in THM response. The correlation between shear strength and thermal effect that it was seemed to decrease stiffness and shear resistance with/without lateral confining pressure. The strength at higher temperature was lower regardless of both with/without lateral confining pressure. Particularly, the thermal effect obviously applied further reduction of strength under saturated condition. Many test results have provided further understanding associate with previous works on THM behavior. The parameters such as stiffness, apparent cohesion and angle of internal friction were measured for safety design specification.

\footnotetext{
* Corresponding author: tomo@ashitech.ac.jp
} 


\section{Testing procedure}

\subsection{Soil materials and summary of specimens}

Kunigel V1 was used in this testing program which was sodium bentonite. Strength of Kunigel V1 was measured as hydro-mechanical properties in previous many works. Having high content of montmorillonite on chemical properties, a bentonite had absolutely fined content larger than $95 \%$. measured density of soil particles was 2.733 $\mathrm{Mg} / \mathrm{m}^{3}$. Also, $\mathrm{SiO}_{2}$ occupied $62 \%$ as chemical components. For mixture, silica sand, named Iitoyo No.4, was used as composing of material, which had highly unique grain size distribution obtained from grain size analysis test. Maximum size was evaluated as $2.0 \mathrm{~mm}$. The mixture ratio of sand and bentonite was 7:3 in dry weight.

Subsequently the bentonite-sand equilibrium to required water content of $17.0 \%$, specimens was compacted statically in the stiffness steel mold, and size of specimens were a diameter of $3.8 \mathrm{~cm}$ and a height of $7.6 \mathrm{~cm}$ with remaining a ratio of 2.0 to diameter against height. Initial specimens had a dry density of 1.600 $\mathrm{Mg} / \mathrm{m}^{3}$, void ratio of 0.710 and degree of saturation of $65.61 \%$ as physical variables. Also, while unsaturated specimen can transfer to saturation in swelling process, the stiffness mold was used as shown in Phot. 1. The all specimen was saturated in acrylic chamber under constant initial volume that vacuum of supply had been performed at least one month in the distilled water. Degree of saturation calculated using water content can prove to be saturation at end of test. Vapor pressure technique was useful to control the high suction, which was over than 40 $\mathrm{MPa}$, and heating performance was conducted to specimen placed in the steel mold as shown in Phot. 2. The mold had was prepared for having specimens and salt water solutions that two salt water solutions were $\mathrm{NaCl}$ and $\mathrm{MgCl}_{2} \cdot 6 \mathrm{H}_{2} \mathrm{O}$. The defined $\mathrm{RH}$ values obtained from using Karlvin's law were $75 \%$ and $33 \%$ at 20 degrees Celsius, relative humidity in the chamber were measured while heating process as following temperature; 20, 40, 60 and 80 degrees Celsius. All the specimen took a free deformation in the chamber at a period of one month that diameter and axial directions permitted to shrinkage or expansion due to changing of soil moisture at equilibrium with each suction. The hyro-mechanical properties for unsaturated-saturated bentonite - sand at various heating temperature are further significant factors, as well as, interpretation of the damages to manmade buffer structure induced by swelling pressure or swelling deformation.

To verify the excess pore pressure of buffer structure composed of compacted bentonite-sand according to heating is necessary to evaluation of safety specification code or simulation mathematical models such as unsaturated soil yield LC curve. Equipment as shown in Phot. 3 was developed which consisted of a stiffness mold, two basement plates and the installed two conventional valves. All the specimen increased the temperature according with heating in the heating controlling chamber, and changing of pore

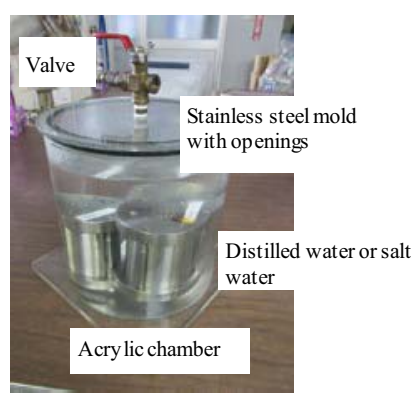

Photo 1. Process saturation using steel mold in chamber.

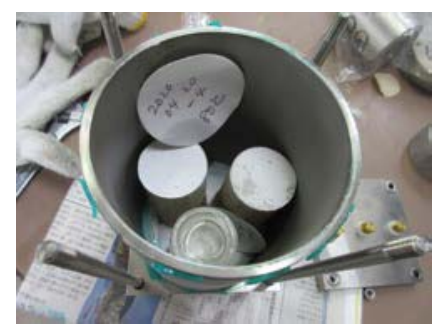

Photo 2. Suction controlling using vapor pressure technique.

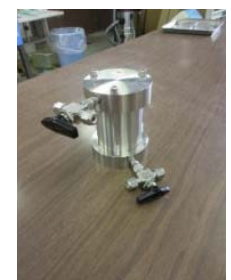

Photo 3. Developed steel mold which is possible to install pressure sensors.

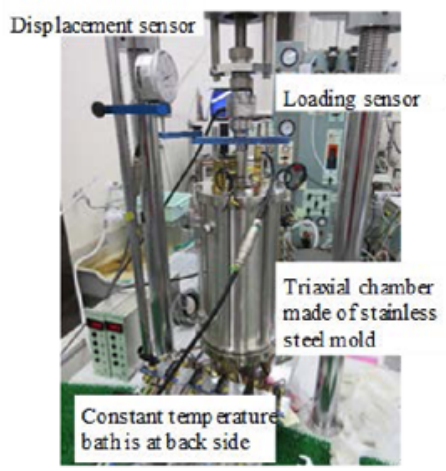

Photo 4. Heating triaxial compression apparatus.

pressure in void structures for bentonite-sand specimen was determined using the pressure transude sensor. The tow pressure sensors were located outside the heating controlling chamber, which had a widely space as $650 \times$ $500 \times 500 \mathrm{in} \mathrm{cm}$. Maximum temperature was up to 200 degrees Celsius.

\subsection{Triaxial apparatus}

A newly-developed thermal triaxial apparatus was used for measurement of unconfined compressive strength of compacted bentonite subjected to thermal impact. The apparatus was indicated as shown in Phot. 2 which can control temperature for isotropic thermal conductivity. The specimen was placed on the pedestal in the inner cell 
made of acrylic material. The inner cell was covered the heating water tube, which was made of stainless steel with a spiral in sharp. The outer chamber was made of stiffness steel material, and had high resistance to heating impact. The measurement of compression stresses was performed using a loading sensor installed in the chamber, which had a resistance to high temperature, and the capacity of maximum temperature was 120 degrees Celsius. Axial deformation was measured using the dial gauge ready installed outside of the outer cell. A membrane with a thickness of $0.5 \mathrm{~mm}$ covered the whole specimen on pedestal. The temperature probe surrounding specimen measured the temperature of heating water supplied in the cell. The water tube was connected to constant temperature control bath. Required temperature that 20 and 80 degrees Celsius were controlled in the constant temperature controlling water bath.

Unconfined compression tests were conducted to evaluate the stress-strain curves with various compression speeds that range from $0.01 \% / \mathrm{min}$ to $1.0 \% / \mathrm{min}$. While the compression process without lateral confining pressure, the temperature was controlled in either 20 or 80 degrees Celsius. The influence of installed membrane used in specimen on shear resistance was assumed to be negligible.

\section{Unconfined compressive strength and pore pressure induced by isotropic heating effort}

The decreasing suction and increment soil moisture lead that shear strength clearly decreases as one of properties in hydro-mechanical phenomena for bentonite. Relationship between compression speed and unconfined compressive strength (after this: strength) was descried in Fig. 1. Unsaturated test data sets were displayed using symbol ( ) that exhibited the largest strength compared to saturated test data sets. According to swell bentonite, it was essentially to delete suction due to both increment of soil moisture and reduction resistance of micro-macro structure. Also, the influence of increment of compression speed created reduction of strength. Unsaturated specimens were applied suction induced by relative humidity as shown in Fig.4. Thermal efforts were due to increasing of temperature in steel mold, and the temperature range performed from 20 degrees to 80 degrees in Celsius. Measured relative humidity was in the steel mold, when $\mathrm{NaCl}$ described namely $70 \%$ in relative humidity, and a relative humidity of $40 \% \mathrm{MgCl}_{2} \cdot 6 \mathrm{H}_{2} \mathrm{O}$ was equilibrium with a relative humidity of $40 \%$. All of the specimens under controlling relative humidity and temperature took some deformations in shrinkage comparison to initial specimen volume that the further efforts caused directly to changing soil moisture and deformation to void structure at macro-micro interface points. Thermal and hydration performance can reveal that shear resistance for compacted, unsaturated bentonite-sand specimens as shown in Fig. 2. The trend obtained from this experimental testing is similar to the interpret as literature on THMC researches for artificial barrier system composing bentonite and sand.
This study concerned pore pressure associated with heating, and it applied the significant stress quantitively changes to the mechanical properties. Using the developed steel mold installed the two pressure sensors, determination of pore pressures was conducted at high temperature, which heating was supplied within isotropic direction. Limited two test results were indicated in several temperatures which were case of 80 degrees and 70 degrees in Celsius. Firstly, 80 degrees Celsius was applied to the specimen over twenty days, and subsequently, it was produced from 80 degrees to 20 degrees in Celsius between ten temperatures, while testing period was exceed ten days at least. Excess fluid pressures composing water and air have not been studied for bentonite, and adequate mathematical simulation models are required to interpret physical properties. This paper exhibited that accurate pore pressures were measured as shown in Figs. 3 and 4. It was further increment when isotropic heating performance began to specimen. The pore pressure approximately reached to $45 \mathrm{kPa}$ for specimen with water content of $17.0 \%$ under 80 degrees, other hand, the pore pressure described $30 \mathrm{kPa}$ for compaction water content of $0.2 \%$. The difference of compaction water content to pore pressure that it related to be the initial degree of saturation associated to volume of air void in void structure. Then, due to decreasing of temperature, measured pore pressure was developed, and indicated the decrement regardless of compaction water content. It was significant reduction that compaction water content of $17.0 \%$ represented approximately 20 $\mathrm{kPa}$.

\section{Influence of temperature to shear strength under confining pressure}

Shear strength under confining pressure was useful to establish quantify parameter that conducted out trial compression test till specimen completely failure.

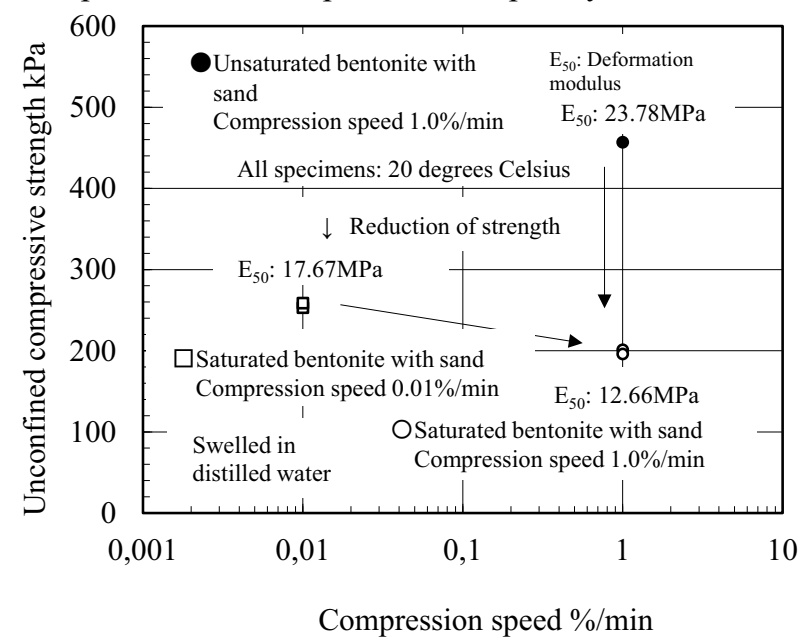

Figure 1. Reduction of unconfined compressive strength due to saturation.

Applied confining pressure was $100 \mathrm{kPa}$ at different temperatures (i.e. a range from 20 degrees and 80 degrees in Celsius). The maximum deviator stress described that shear resistance was peak when a temperature was 20 
degrees regardless of unsaturation or saturation. All of the unsaturated specimens indicated smooth reduction according to increment of temperature and it was approximately linear line. Saturation specimen can seem the comparable trend to unsaturated specimens. The decreasing of maximum deviator stress for saturation was slightly larger than that of unsaturated condition when temperature was over 40 degrees. Although many reports verified that reduction of shear strength due to heating was common in the experimental works, this study is in comparison of unsaturated condition and saturated condition.

\section{Conclusions}

The study represented the influence of thermal-hydro efforts on strength and occurrence of pore pressure for bentonite-sand materials. Summaries were described as following. It was proposed that hydration effort due to soil moisture increment caused the reduction of shear resistance, and the study considered the changing unconfined compressive strength. Then, the influence of temperature was further significant to shear strength for unsaturated bentonite-sand.

It was obviously in advantage comprehensive results that pore pressure has grown rapidly at beginning of heating application, which was isotropic conductivity. Remaining occurrence in excess pressure was collected following to increasing temperature.

It was can be determined maximum deviator stress at confining pressure of $100 \mathrm{kPa}$ with various temperatures in triaxial compression test. Shear strength indicated further decreasing beyond heating performance.

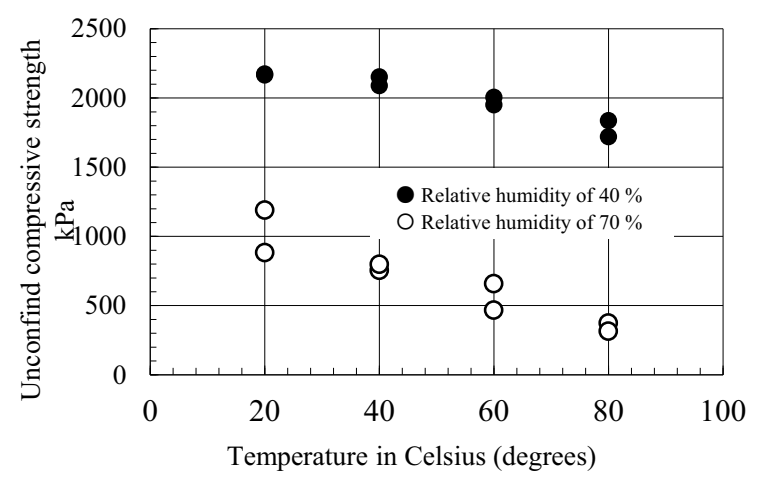

Figure 2. Decreasing of unconfined compressive strength according to heating for tow difference relative humidity.

\section{References}

1. P. Delage, Y. J. Cui \& A. M. Tang. (2010). Clays in radioactive waste disposal, Journal of Rock Mechanics and Geotechnical Engineering, 2(2), 111123.

2. P.Wersin, L.H.Johnson, \& I.G.McKinley. (2007): Performance of the bentonite barrer at temperatures beyond $100{ }^{\circ} \mathrm{C}$ : A critical review, Physics and Chemistry of the Earth, Parts A/B/C, 32(8), 780-788
3. M. Åkesson, A. C. Jacinto, C. Gatabin, M. Sanchez, \& A. Ledesma. (2009). Bentonite THM behaviour at high temperatures: experimental and numerical analysis, Géotechnique, 59, 4, 307-318.

4. A.M. Tang \& Y.J. Cui. (2005). Controlling suction by the vapour equilibrium technique at different temperatures and its application in determining the water retention properties of MX-80 clay. Can. Geotech. J, 42, 1, 287-296.

5. M.V. Villar \& A. Lloret. (2004). Influence of temperature on the hydro-mechanical behaviour of a compacted bentonite. Appl. Clay Sci, 26, 1-4, 337350 .

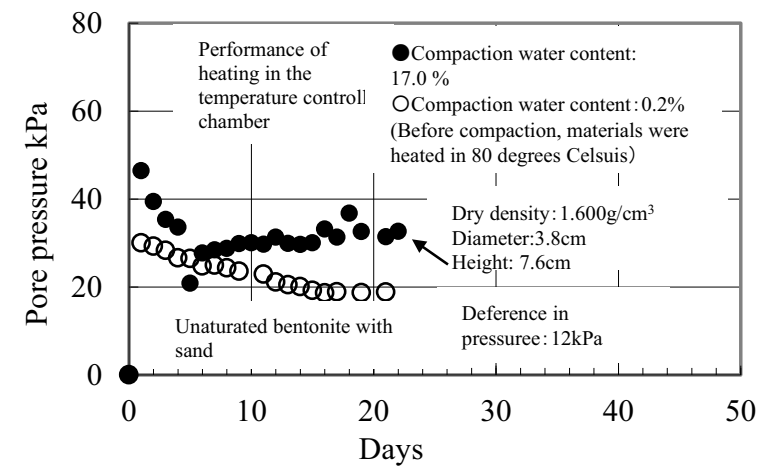

Figure 3. Occurrence of pore pressure in unsaturated specimen under 80 degrees Celsius.

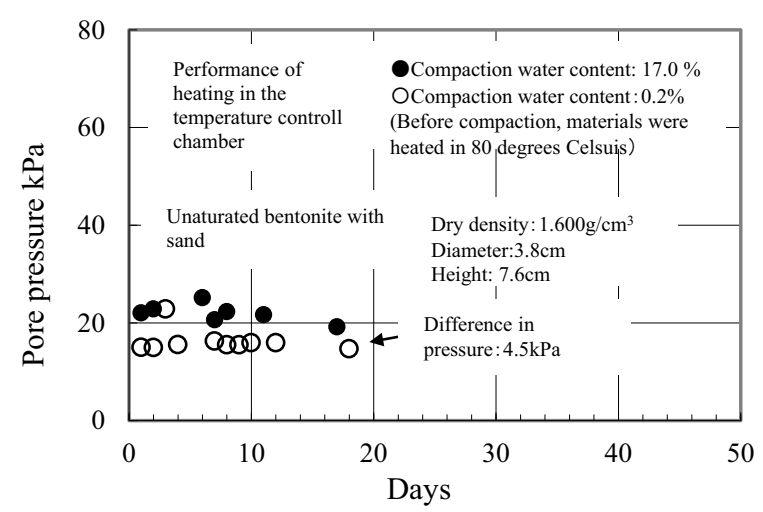

Figure 4. Occurrence of pore pressure in unsaturated specimen under 70 degrees Celsius.

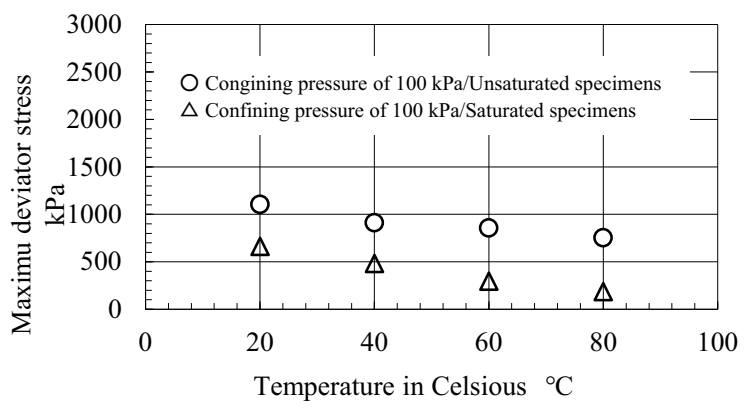

Figure 5. Reduction of maximum deviator stress for unsaturated/saturated specimens with temperature. 